\title{
Nurses' Behavior Regarding Pain Treatment in an Emergency Department: A Single-Center Observational Study
}

This article was published in the following Dove Press journal:

Journal of Pain Research

\author{
Salvatore Sardo $\mathbb{D}^{1,2}$ \\ Maura Galletta $\mathbb{D i D}^{2}$ \\ Erica Coni ${ }^{3}$ \\ Cesar Ivan Aviles Gonzalez (D) \\ llenia Piras (iD ${ }^{4}$ \\ Giorgio Pia ${ }^{4}$ \\ Maurizio Evangelista $\mathbb{D}^{5}$ \\ Mario Musu (D) ${ }^{1,2}$ \\ Gabriele Finco (D) ${ }^{1,2}$
}

\footnotetext{
'Anesthesia and Intensive Care Unit, University of Cagliari, Cagliari, Italy; ${ }^{2}$ Department of Medical Sciences and Public Health, University of Cagliari, Cagliari, Italy; ${ }^{3}$ RN-BC, University of Cagliari, Cagliari, Italy; ${ }^{4}$ ATS Sardegna, ASSL Cagliari SS, Cagliari, Italy; ${ }^{5}$ Department of Anesthesiology and Pain Medicine, Cattolica University, Rome, Italy
}

Purpose: The aim of this prospective study was to assess the behavior of emergency department (ED) nurses with regard to pain and their role in pain management in a reallife clinical setting.

Methods: A total of 509 consecutive patients were enrolled during a 6-week period. A casereport form was used to collect data on nurses' approaches to pain, time to analgesia provision, and patient-perceived quality of analgesia.

Results: Triage nurses actively inquired about pain in almost every case, but they did not estimate pain intensity in a third of patients. In the majority of cases, triage nurses did not report pain-related findings to the physician, who was the only professional that could prescribe analgesia to patients. The assignment of the color-coding of triage by nurses appears to be related to the perceived severity of the clinical case and a more comprehensive evaluation of pain. More than half of patients were at least fairly satisfied with analgesia.

Conclusion: Pain is increasingly screened during triage, but its comprehensive assessment and management still lack systematic application. We believe that further education and implementation of analgesia protocols may empower nurses to manage ED patients' pain more effectively and in a more timely manner.

Keywords: emergency, ED, nurse, oligoanalgesia, pain, triage

\section{Introduction}

The need for a cultural shift in Italian health professionals' behavior regarding pain was reflected in the promulgation of law 38/2010. This Italian law requires mandatory evaluation, management, and tracking of pain intensity in health records. ${ }^{1}$ Despite this law, pain undertreatment remains common. In 1989, the term "oligoanalgesia" was coined in reference to the poor management of pain in emergency departments (EDs), which is still a ubiquitous problem. ${ }^{2}$ Indeed, the great majority of patients present to EDs with pain as their chief complaint and receive unsatisfactory analgesia. ${ }^{2-6}$ Our previous research showed that $32.46 \%$ of patients complaining of pain at a second-level urban ED did not receive analgesia, while 11.48\% left the ED before clinical examination. $^{7}$

In view of the long waiting time before physician examinations, we considered the triage nurse as the first health professional who may evaluate and manage ED patients' pain. Nurse-led triage was associated with improved analgesia in a nationwide prospective study on 50 French EDs. ${ }^{8}$ Indeed, in a recent literature review, Hatherley et al put forward five recommendations to improve acute-pain
Correspondence: Maura Galletta Department of Medical Sciences and Public Health, University of Cagliari, Highway 554 (exit for Sestu), Monserrato 09042, Cagliari, Italy

Tel +39 070-675-3108

Email maura.galletta@gmail.com 
management: mandating pain scores, offering or administering analgesia within 30 minutes, using time to analgesia as an ED-performance indicator, reassessing pain after treatment, and implementing nurse-led analgesia protocols. ${ }^{9}$ In order to clarify behavior with regard to pain and performance of triage nurses, we designed this single-center observational study.

\section{Methods}

The authors designed and performed this prospective observational study in accordance with the Good Clinical Practice (GPC) guidelines, the EU CT Directive 2001/20/ EC, the GCP Commission Directive 2005/28/EC, and the Declaration of Helsinki (as it stood in 2008). This study was referred to the Independent Ethical Committee of Cagliari Teaching Hospital, which approved it as a lowintervention observational study (PROT.PG/2018/17,157). The committee reviewed the study and established an exemption from inclusion in the National Observational Study Register.

A simple case-report form (CRF) was designed to describe the behavior of triage nurses in terms of pain management in the ED (see Attachment 1). The CRF had the primary aim of evaluating ED nurses' behavior pertaining to pain. Secondary aims were to describe timing, modality, and patients' perception of ED analgesia. Nurse behavior, timing, and modality (items 2-10) were assessed via direct observation by the researchers, who followed patients during all triage phases until discharge/transfer. To measure patient perceptions about ED analgesia (items 11-13), the researchers interviewed patients directly prior to discharge or transfer to another department. Moreover, patients' medical records were also consulted to collect information about pain sites. The authors chose a secondlevel urban ED (Santissima Trinità Hospital, ATS Sardegna, Cagliari, Italy) to collect data of consecutive patients during the day shift (07 am-10 pm) over a 6-week period. Data were collected from May 8th to June 5th, 2018. Inclusion criteria were ability to provide valid consent for patients aged $\geq 18$ years or proxy consent for minors and triage registration. Exclusion criteria included refusal or inability to give consent to the study procedures (including coma, psychomotor agitation, and drug intoxication). At the end of June, survey data were manually transferred from paper to an electronic spreadsheet (Excel; Microsoft, Redmond, WA, USA) by the researchers who collected them. Patients' anonymity and confidentiality were assured by virtue of the fact that the
CRF was depersonalized and coded. Analysis of stored data was performed with SPSS version 20.0 (IBM, Armonk, NY< USA) and tables were plotted with Excel.

\section{Results}

During the sampling period (May-June 2018), 266 male patients and 243 female patients (total sample size of 509), were enrolled, and all of them complained of acute pain. The mean age was $48.46 \pm 24.36$ years. Two patients left the ED before receiving any treatment. Triage nurses inquired about pain presence in $95 \%$ of cases, but evaluated pain intensity in a smaller proportion of patients (69.7\%), using in every instance the numeric rating scale (NRS). Nurse-perceived severity of case was expressed by the triage color code related to pain-intensity assessment.

Triage nurses did not evaluate pain intensity in $48.5 \%$ of white-coded (no urgency) and $36.2 \%$ of green-coded (minor urgency) patients, while they missed this estimation in only $19.6 \%$ of yellow-coded (delayed urgency), and no red-coded patients (emergency; Table 1). In the vast majority of cases, $89.2 \%$, nurses implicitly associated pain-assessment priority with urgency for ED admission. Nurses did not inform physicians about patients' pain in $84.1 \%$ of cases, and they never managed pain autonomously during triage. Only $38.5 \%$ of patients received analgesia within 60 minutes after triage (Table 2). Patientreported reduction in pain intensity on the NRS was 3

Table I Assessment of Pain and Triage Color Codes

\begin{tabular}{|c|c|c|c|c|}
\hline & & \multicolumn{2}{|c|}{ Nurse's Inquiries about Pain } & \multirow[t]{2}{*}{ Total } \\
\hline & & Yes & No & \\
\hline \multirow[t]{4}{*}{ Triage code } & White & 30 & 3 & 33 \\
\hline & Green & 255 & 19 & 274 \\
\hline & Yellow & 194 & 5 & 199 \\
\hline & Red & 3 & 0 & 3 \\
\hline \multirow{3}{*}{\multicolumn{2}{|c|}{ Total }} & 482 & 27 & 509 \\
\hline & & \multicolumn{2}{|c|}{$\begin{array}{l}\text { Nurse's Evaluation of Pain } \\
\text { Intensity }\end{array}$} & \multirow[t]{2}{*}{ Tota } \\
\hline & & Yes & No & \\
\hline \multirow[t]{4}{*}{ Triage code } & White & 17 & 16 & 33 \\
\hline & Green & 175 & 99 & 274 \\
\hline & Yellow & 160 & 39 & 199 \\
\hline & Red & 3 & 0 & 3 \\
\hline \multicolumn{2}{|l|}{ Total } & 355 & 154 & 509 \\
\hline
\end{tabular}

Notes: White, no urgency; green, minor urgency; yellow, delayed urgency; red, emergency or resuscitation. 
Table 2 Time from ED Triage to Nurse-Administered Analgesia

\begin{tabular}{|l|l|l|l|}
\hline & Frequency & $\%$ & Cumulative \% \\
\hline Lost to follow-up & 2 & 0.4 & 0.4 \\
Immediate & 7 & 1.4 & 1.8 \\
I-30 minutes & 187 & 36.7 & 38.5 \\
$31-60$ minutes & 82 & 16.1 & 54.6 \\
$61-90$ minutes & 57 & 11.2 & 65.8 \\
$91-150$ minutes & 85 & 16.7 & 82.5 \\
I5I-240 minutes & 54 & 10.6 & 93.1 \\
$4-7$ hours & 28 & 5.5 & 98.6 \\
$>7$ hours & 7 & 1.4 & 100 \\
\hline Total & 509 & 100 & - \\
\hline
\end{tabular}

Table 3 Decrease in Pain Intensity after Analgesia Administration

\begin{tabular}{|l|l|l|l|}
\hline Decrease in NRS & Frequency & $\%$ & Cumulative \% \\
\hline Lost to follow-up & 2 & 0.4 & 0.4 \\
I point & 52 & 10.2 & 10.6 \\
3 points & 247 & 48.5 & 59.1 \\
5 points & 174 & 34.2 & 93.3 \\
$>5$ points & 34 & 6.7 & 100 \\
\hline Total & 509 & 100 & - \\
\hline
\end{tabular}

Abbreviation: NRS, numeric rating scale.

points and 5 points in $48.6 \%$ and $33.6 \%$ of cases, respectively (Table 3 ).

The percentage of patients who perceived the quality of analgesia as fair and good was $49.5 \%$ and $20.6 \%$, respectively, while $0.4 \%$ considered it poor. Only $2 \%$ of the sample considered it excellent. Absolute decrease in pain intensity (NRS) was deemed more important than time to analgesia in determining the perceived quality of analgesia (Table 4): $84 \%$ of patients who judged analgesia as good experienced a 5-point reduction in pain intensity, while $57 \%$ who rated analgesia as fair had a 3-point NRS-score reduction. The site of pain (Table 5) did not appear to be related to time to analgesia, but the study was not designed to assess this outcome. As shown in Table 6, nurse
Table 5 Sites of Pain

\begin{tabular}{|l|l|l|}
\hline & $\mathbf{n}$ & $\%$ \\
\hline Head & 82 & 16.1 \\
Neck & 38 & 7.5 \\
Chest & 75 & 14.7 \\
Abdomen & 67 & 13.2 \\
Pelvic area & 50 & 9.8 \\
Lumbosacral spine & 24 & 4.7 \\
Upper limbs & 79 & 15.5 \\
Lower limbs & 72 & 14.1 \\
Burns or superficial injuries & 22 & 4.3 \\
\hline Total & 509 & 100 \\
\hline
\end{tabular}

Table 6 Sites of Pain and Nurse Evaluation of Pain Intensity

\begin{tabular}{|l|l|l|l|l|}
\hline \multirow{2}{*}{} & \multicolumn{3}{l}{ Nurse Evaluation } \\
\cline { 2 - 5 } & \multicolumn{2}{|l|}{ Yes } & \multicolumn{2}{l|}{ No } \\
\cline { 2 - 5 } & n & $\%$ & n & $\%$ \\
\hline Head & 62 & 17.5 & 20 & 13.0 \\
Neck & 26 & 7.3 & 12 & 7.8 \\
Chest & 60 & 16.9 & 15 & 9.7 \\
Abdomen & 50 & 14.1 & 17 & 11.0 \\
Pelvic area & 33 & 9.3 & 17 & 11.0 \\
Lumbosacral spine & 15 & 4.2 & 9 & 5.8 \\
Upper limbs & 49 & 13.8 & 30 & 19.5 \\
Lower limbs & 51 & 14.4 & 21 & 13.6 \\
Burns or superficial injuries & 9 & 2.5 & 13 & 8.4 \\
\hline
\end{tabular}

Notes: $\chi^{2}=17.73 ; d f=8 ; p<0.05$.

evaluation of pain intensity during triage was mainly performed with patients presenting with head $(17.5 \%)$, chest $(16.9 \%)$, and abdomen (14.1\%) pain, while in patients who did not receive a pain-intensity evaluation sites of pain were upper limbs (19.5\%) and the skin $\left(8.4 \%, \chi^{2}=17.73, d f\right.$ $=8 ; p<0.05)$.

\section{Discussion}

The prevalence of pain in our cohort was higher than that reported in our previous work carried out in the same clinical

Table 4 Decrease in NRS Score and Perceived Quality of Analgesia

\begin{tabular}{|c|c|c|c|c|c|c|c|}
\hline & & \multicolumn{5}{|c|}{ Patient-Perceived Quality of Analgesia } & \multirow[t]{2}{*}{ Total } \\
\hline & & Insufficient & Sufficient & Fair & Good & Excellent & \\
\hline \multirow[t]{2}{*}{ Pain-intensity decrease } & $\leq 3$ points & 2 & 137 & 143 & 17 & 0 & 299 \\
\hline & $\geq 5$ points & 0 & 1 & 109 & 88 & 10 & 208 \\
\hline \multicolumn{2}{|l|}{ Total } & 2 & 138 & 252 & 105 & 10 & 507 \\
\hline
\end{tabular}

Abbreviation: NRS, numeric rating scale. 
setting $(70.7 \%)$ and in the published literature. ${ }^{3,5,7,10}$ In this work, ED nurses demonstrated attention to pain and actively inquired about pain presence in almost every case. This may explain such a high pain prevalence. Similar results were shown in a recent study, in which a high percentage of nurses (69\%) pursued patients' relief from pain. This result was explained by their knowledge of and attitudes toward pain management. ${ }^{11}$ Also, in our study nurses had a proactive approach toward pain detection, but they missed the critical step of evaluating pain intensity in a significant proportion of patients $(30.3 \%)$. This may be related to both frustration and burnout or to poor knowledge of the importance of performing a correct pain assessment aimed at establishing adequate pain treatment.

Furthermore, nurses' approaches in terms of pain appeared to be influenced by severity-of-illness bias, as reported in the diverse comprehensive pain evaluations expressed by the triage color code. This finding suggests that nurses consider pain a symptom and pain intensity proportional to the severity of a disease. Consequently, ED nurses may fail to detect and address a pain syndrome manifestly unrelated to a severe or critical disease. There was homogeneity regarding the evaluation tool for pain intensity, the NRS, showing its usefulness and immediacy in the time-pressured environment of the ED. No nurse administered analgesic drugs autonomously, and in the vast majority of cases, pain was not mentioned in nursephysician communication.

Traditional barriers to pain management in the ED are related to fear of compromising the medical diagnostic process by providing early analgesia, especially for visceral pain. ${ }^{12}$ Indeed, in our study physician had the responsibility of authorizing drug administration, and thus analgesia was delayed from the beginning of the diagnostic process. In order to reduce patient suffering in the ED, a protocol for pharmacological treatment for acute pain would be useful. This would reduce patient waiting times for analgesic treatment and pain-related complications, as highlighted by recent European guidelines. ${ }^{13}$ Also, the literature emphasizes the importance to nurses to treat pain in accordance with evidence-based practice. Nevertheless, as shown by a recent Polish study, a main barrier for nurses could be the fact that the scientific literature is mainly in English. ${ }^{14}$ This barrier may also involve Italian nurses. In this study, patient-perceived quality related more to the decrease in pain intensity rather than time to analgesia. This finding emphasizes the importance of pain-intensity estimation using validated scales, in order to choose appropriate first-line treatment.

\section{Limitations}

The limitations of our study lie in the single-center design and the day shift-only sampling, but these features may not jeopardize the generalizability of our findings, because we chose the busiest time in the ED and a real-life clinical setting. Additionally, we chose the same center as our previous work on pain prevalence, so we had already investigated its epidemiology of pain. ${ }^{7}$ Moreover, the sampling period was quite short, but it was deemed to be sufficient to provide us with a snapshot of nurses' roles and behavior with regard to pain management in a nonacademic clinical environment. Finally, this study focused on nurses' behaviors and actions in terms of pain management without a psychometric investigation of their attitudes toward pain, which may affect pain management. ${ }^{11}$ Therefore, it is likely that this aspect may have influenced triage nurses' performance in this setting. A future direction of study could include assessment of nurses' attitudes as a possible cause of persistent oligoanalgesia in the ED setting.

\section{Conclusion}

In the published literature, the role of nurses in terms of attitudes and behavior regarding pain is being increasingly investigated, because it affects pain management in health care. A recent cross-sectional survey-based study on 696 Italian nurses showed that patterns of practice and approaches to pain are heterogeneous across Italy, as demonstrated by a less proactive management of pain and less knowledge of pain scales and structured questionnaires in southern regions. ${ }^{13}$

Our findings support the thesis that, during triage, pain is often overlooked. Barriers to better pain management could be related to both cultural and organizational factors. Specific barriers in nurses' work may include both insufficient time spent with patients and ignorance of patients' ability to tolerate pain intensity. ${ }^{15}$ Moreover, an incomplete implementation of the current Italian law (n.38/2010), as well as recommendations from Italian scientific societies, ${ }^{16}$ could represent other barriers to managing pain in the ED.

The implementation of an ED analgesia protocol may empower nurses to reduce the long patient waiting time for analgesia. Indeed, this topic has been investigated, with encouraging results regarding both time and effectiveness of analgesia. ${ }^{17,18}$ In order to do that, nurses and physicians should develop shared internal protocols that guarantee prompt recognition and management of pain through standards used in the ED. Lastly, more education is required to 
overcome some cultural bias on pain assessment in the ED setting.

\section{Author Contributions}

All authors made a significant contribution to the work reported, whether that is in the conception, study design, execution, acquisition of data, analysis and interpretation, or in all these areas; took part in drafting, revising or critically reviewing the article; gave final approval of the version to be published; have agreed on the journal to which the article has been submitted; and agree to be accountable for all aspects of the work.

\section{Funding}

This research did not receive any specific grant from funding agencies in the public, commercial, or not-forprofit sectors. No sponsor(s) were involved in this project.

\section{Disclosure}

The authors report no conflicts of interest in this work.

\section{References}

1. Ripamonti C, Valle A, Peruselli C, Pessi MA, Prandi C. [Project "Hospital without pain": analysis of the Italian situation before the law 38]. Assist Inferm E Ric AIR. 2011;30(2):95-99. doi:10.1702/845.9395. Italian.

2. Wilson JE, Pendleton JM. Oligoanalgesia in the emergency department. Am J Emerg Med. 1989;7(6):620-623. doi:10.1016/ 0735-6757(89)90286-6

3. Tanabe P, Buschmann M. A prospective study of ED pain management practices and the patient's perspective. J Emerg Nurs JEN off Publ Emerg Dep Nurses Assoc. 1999;25(3):171-177. doi:10.1016/S00991767(99)70200-X

4. Dale J, Bjørnsen LP. Assessment of pain in a Norwegian emergency department. Scand J Trauma Resusc Emerg Med. 2015;23:86. doi:10.1186/s13049-015-0166-3

5. Johnston CC, Gagnon AJ, Fullerton L, Common C, Ladores M, Forlini S. One-week survey of pain intensity on admission to and discharge from the emergency department: a pilot study. $J$ Emerg Med. 1998;16(3):377-382. doi:10.1016/S0736-4679(98)00012-2

6. Todd KH, Ducharme J, Choiniere M, et al. Pain in the emergency department: results of the pain and emergency medicine initiative (PEMI) multicenter study. $J$ Pain off $J$ Am Pain Soc. 2007;8 (6):460-466. doi:10.1016/j.jpain.2006.12.005
7. Mura P, Serra E, Marinangeli F, et al. Prospective study on prevalence, intensity, type, and therapy of acute pain in a second-level urban emergency department. J Pain Res. 2017;10:2781-2788. doi:10.2147/JPR.S137992

8. Guéant S, Taleb A, Borel-Kühner J, et al. Quality of pain management in the emergency department: results of a multicentre prospective study. Eur J Anaesthesiol. 2011;28(2):97-105. doi:10.1097/ EJA.0b013e $3283418 \mathrm{fb} 0$

9. Hatherley C, Jennings N, Cross R. Time to analgesia and pain score documentation best practice standards for the emergency department - a literature review. Australas Emerg Nurs J AENJ. 2016;19 (1):26-36. doi:10.1016/j.aenj.2015.11.001

10. Tcherny-Lessenot S, Karwowski-Soulié F, Lamarche-Vadel A, Ginsburg C, Brunet F, Vidal-Trecan G. Management and relief of pain in an emergency department from the adult patients' perspective. J Pain Symptom Manage. 2003;25(6):539-546. doi:10.1016/S08853924(03)00147-7

11. Bashar IA, Nor Azimah CA. Pain management practices by nurse: an application of the knowledge, attitude, and practices (KAP) model. Glob J Health Sci. 2016;8:154-160. doi:10.5539/gjhs.v8n6p154

12. Wolfe JM, Lein DY, Lenkoski K, Smithline HA. Analgesic administration to patients with an acute abdomen: a survey of emergency medicine physicians. Am J Emerg Med. 2000;18(3):250-253. doi:10.1016/S0735-6757(00)90114-1

13. Angeletti C, Guetti C, Paesani M, Colavincenzo S, Ciccozzi A, Angeletti PM. An analysis of Italian nurses' approach to patients' pain: a nationwide online survey. Pain Res Manag. 2018;2018:5165262. doi:10.1155/2018/5165262

14. Mędrzycka-Dąbrowska W, Dąbrowski S, Gutysz-Wojnicka A, Basiński A. Polish nurses' perceived barriers in using evidencebased practice in pain management. Int Nurs Rev. 2016;63 (3):316-327. doi:10.1111/inr.12255

15. Mędrzycka-Dąbrowska WA, Dąbrowski S, Basiński A, Pilch D. Perception of barriers to postoperative pain management in elderly patients in Polish hospitals with and without a "Hospital Without Pain" Certificate - a multi-center study. Arch Med Sci. 2016;12 (4):808-818. doi:10.5114/aoms.2015.54768

16. Savoia G, Coluzzi F, Di Maria C, et al. Italian intersociety recommendations on pain management in the emergency setting (SIAARTI, SIMEU, SIS 118, AISD, SIARED, SICUT, IRC). Minerva Anestesiol. 2015;81(2):205-225.

17. Ridderikhof ML, Schyns FJ, Schep NW, Lirk P, Hollmann MW, Goslings JC. Emergency department pain management in adult patients with traumatic injuries before and after implementation of a nurse-initiated pain treatment protocol utilizing fentanyl for severe pain. J Emerg Med. 2017;52(4):417-425. doi:10.1016/j.jemermed. 2016.07.015

18. Butti L, Bierti O, Lanfrit R, et al. Evaluation of the effectiveness and efficiency of the triage emergency department nursing protocol for the management of pain. J Pain Res. 2017;10:2479-2488. doi:10.2147/JPR.S138850
Journal of Pain Research

\section{Publish your work in this journal}

The Journal of Pain Research is an international, peer reviewed, open access, online journal that welcomes laboratory and clinical findings in the fields of pain research and the prevention and management of pain. Original research, reviews, symposium reports, hypothesis formation and commentaries are all considered for publication. The manuscript

Submit your manuscript here: https://www.dovepress.com/journal-of-pain-research-journa management system is completely online and includes a very quick and fair peer-review system, which is all easy to use. Visit http:// www.dovepress.com/testimonials.php to read real quotes from published authors. 